\title{
Contact Characteristics Analysis of Taper Rollers and Oilstones in Through-feed Superfinishing
}

\author{
Xue Jinxue $^{1 \mathrm{a}}$, Yang Baisong ${ }^{1 \mathrm{~b}}$, Li Zhuoying ${ }^{2 \mathrm{c}}$ \\ ${ }^{1}$ Henan University of Science and Technology, School of Mechanical and Electrical Engineering, \\ Luoyang Henan China \\ ${ }^{2}$ Jilin University, School of Mechanical Science and Engineering, Changchun Jilin China \\ axjx19652000@163.com, ${ }^{b} 575799150 @ q q . c o m, ~ c$ sanyehuakai@163.com
}

Keywords: Taper rollers; Oilstones; Through-feed superfinishing; Radial contact arc length; Dynamic valid cutting edge numbers; Cutting surface depth

\begin{abstract}
The regularity of the through-feed superfinishing of the taper roller is considered, the radial contact arc length between a oilstone and a roller is calculated, the model of superfinishing dynamic valid cutting edge numbers is built, the influence of key parameters to radial contact arc length and dynamic valid cutting edge numbers is analyzed. The result is given as follows. The two parameters that keep the greatest influence on the arc length and the cutting edge numbers are the roller diameter and the cutting surface depth of the oilstone. With increase of the two parameters in the big end of the roller, the arc length and the cutting edge numbers increase rapidly. The roller generatrix can be machined into perfect crown shape by changing cutting surface depth reasonably and controlling the distribution of dynamic valid cutting edge along the axial direction.
\end{abstract}

\section{Introduction}

The taper roller is a key component of rolling bearings. The shape of the roller crown is important to roller quality[1]. Both theoretical analysis and experimental verification have proven that logarithmic crown is perfect[2]. The logarithmic crown has a positive effect on improving contact stress distribution of rollers and raceways and it is important for improving quality and life of rolling bearings[3,4]. Superfinishing is the most important process of machining roller crown[5]. Through-feed superfinishing is the main machining method of the roller. Yet formation mechanism of roller crown is not clear on theory. The distribution condition of oilstone cutting edge is an important factor to influence material removal rate of the roller. This paper draw lessons from modeling methods of grinding wheel, bases on experimental data and parameter, analyse contact surface shape in superfinishing area and distribution of oilstone cutting edge.

\section{The Processing Method of the Through-feed Superfinishing}

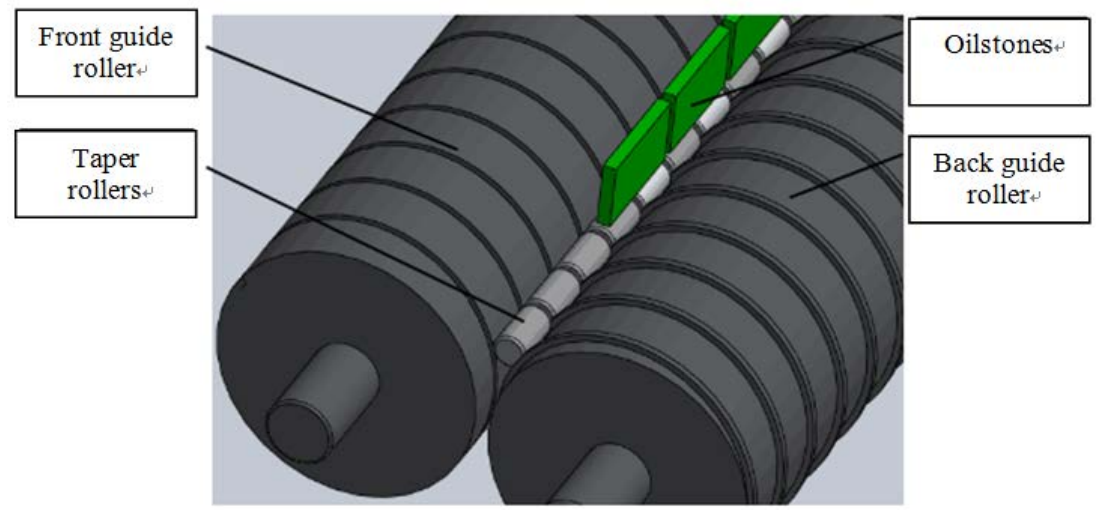

Fig.1 Machining method sketch map of through-feed superfinishing of taper rollers

Fig. 1 shows the processing method of through-feed superfinishing of taper rollers by oilstones. Two guide rollers rotate in the fixed axis and the same direction. Rollers move along the axial and 
rotate by the driving force of the two guide rollers. A row of oilstones press on the rollers with appropriate pressures and oscillate back and forth along the axial. This paper regard the taper roller as one that many thin slices stack up, and the radius of these thin slices are from small to large.

\section{Analysis of the Radial Contact Arc Length}

Fig. 2 shows the geometry relationship between oilstones and rollers. Fig. a is axial main view and Fig. b is radial left view. $r_{w}$ represent roller radius, $r_{s}$ represent contact surface radius. $\psi$ represent contact angle. $\Delta z$ represents width of a roller thin slice. Fig. a shows that the superfinishing speed of abrasives equal rotational speed of rollers. Fig. b shows that the superfinishing speed of abrasives equal superpose speed of oscillation speed of oilstones and axial speed of rollers.

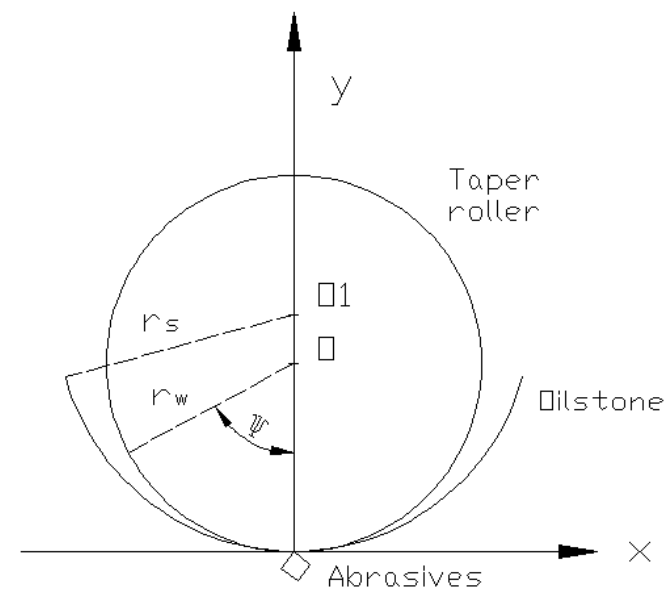

(a)Axial main view

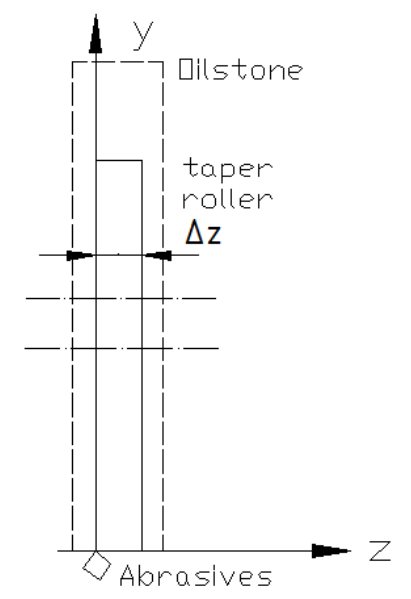

(b) radial left view

Fig.2 Contact sketch map of rollers and olstones

Based on the analysis above, the part of particle equation of abrasives is as follows.

$$
\left\{\begin{array}{c}
x=r_{w} \sin \psi \\
y=r_{w}(1-\cos \psi) \\
z=\frac{\psi}{2 \pi \frac{n_{w}}{60}}\left(v_{s} \pm f_{a}\right) \\
v_{s}=4 A f
\end{array}\right.
$$

$n_{W}$ represents rotational speed of rollers, $V_{S}$ represents oscillation average speed of oilstones, $A$ represents amplitude of oilstones, $f$ represents oscillation frequency of oilstones, $f_{a}$ represents axial feed rate of rollers. It is “-” when the direction of oilstone oscillation and the direction of roller axial feed are the same, and is "+" when contrary.

$$
\left\{\begin{aligned}
1_{s}=\int_{0}^{\psi} d l_{s} & =\psi \sqrt{r_{W}^{2}+\left[\frac{30}{\pi n_{W}}\left(V_{s} \pm f_{a}\right)\right]^{2}} \\
\psi & \approx 2 \sqrt{\frac{d_{S} a_{S}}{d_{W}\left(d_{s}-d_{W}\right)}} \\
d_{e} & =\frac{d_{s}}{d_{W}\left(d_{s}-d_{W}\right)}
\end{aligned}\right.
$$


Integrate the equation $d l_{s}=\sqrt{d x^{2}+d y^{2}+d z^{2}}$ and obtain the equation of $l_{s} \cdot d_{s}$ represents the superfinishing diameter of oilstones, $d_{w}$ represents roller diameter.

Fig. 3 shows $\psi, a_{s}$ represents cutting surface depth of oilstones. Many factors like radial pressure, contact area, material properties of oilstones and rollers decide the value of $a_{s}$ which can be obtained by experiments.

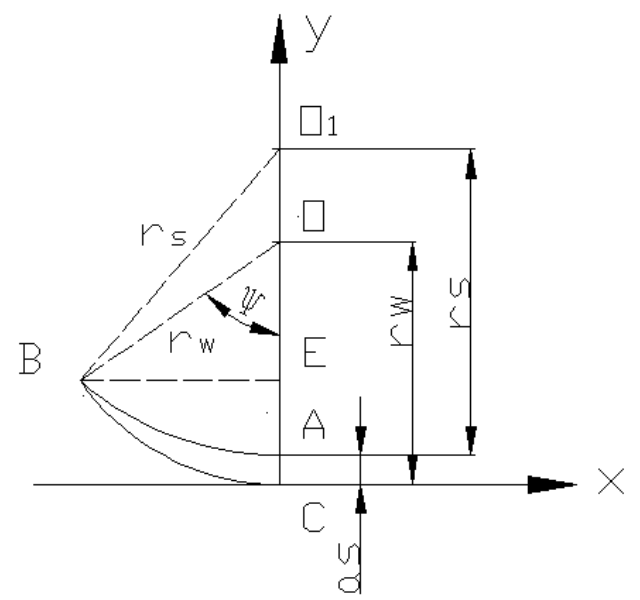

Fig. 3 contact angle sketch map of rollers and oilstones

Actual radial contact arc length is 1.3 times to 2.3 times longer than theory[6]. This paper add a coefficient $k_{t}$ which is bigger than 1 and its value can be obtained by experiments. The influence of $f_{a}$ to $l_{s}$ can be ignored. According to conclusions above, the equation of $l_{s}$ is as follows.

$$
I_{s}=k_{t} \sqrt{d_{e} a_{s}} \cdot \sqrt{d_{W}^{2}+\left(\frac{60 v_{s}}{\pi n_{W}}\right)^{2}}
$$

According to references[6,7], the key parameters in Eq. 3 can be determined as followed: $k_{t}=1.3, A=1 \mathrm{~mm}, f=45 \mathrm{cycles} / \mathrm{s}, d_{s}=10 \mathrm{~mm}, 8 \mathrm{~mm} \leq d_{w} \leq 10 \mathrm{~mm}, a_{s}=0.005 \mathrm{~mm}$, $n_{w}=600 \mathrm{r} / \mathrm{min}$. Fig. 4 shows the result of MATLAB.

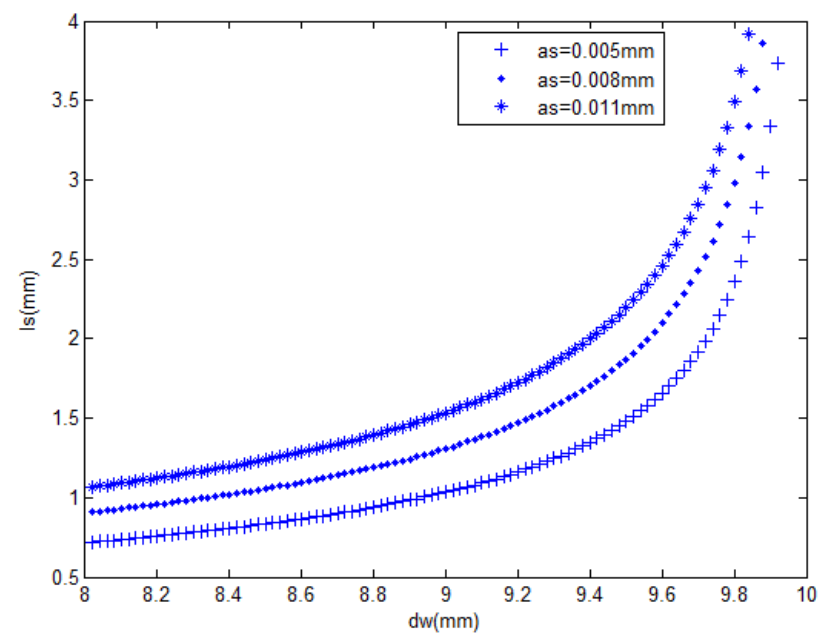

Fig.4 The influence of $a_{s}$ to $l_{s}$

What can be founded by observing the various curves in Fig. 4 is that radial contact arc length $l_{s}$ increases with the increase of tapered roller diameter $d_{w}$. From the small end of the roller to the middle portion it increases slowly, at the large end it increases quickly. This phenomenon can be 
explained as follows. First, with the increase of $d_{w}$, the roller shape of the superfinishing part and the surface shape of the oilstone become more and more similar. Second, with the increase of $d_{w}$, the relative velocity of the roller and the oilstone increases constantly. It is already proved in the grinding model that the bigger the relative velocity is, the smaller the contact area is. The superfinishing contact surface shaped like a " $\pi$ ", which is in line with expectations.

The radial contact arc length $l_{s}$ increases with the increase of machining surface depth $a_{s}$ when $d_{w}$ is the same, that means $a_{s}$ has a great influence on $l_{s}$. This conclusion is entirely consistent in the actual situation. It can be seen from the distance between the adjacent two curves that, the increase speed of $l_{s}$ with the increase of $a_{s}$ remains substantially unchanged.

\section{Calculation and Analysis of Cutting Edge Numbers}

On the surface of the oilstone, the height of abrasive is not the same. Determine a cutting surface depth value $a_{s}$ along radial of oilstone surface, and the conclusion can be obtained that only a part of abrasives can participate in superfinishing. The calculating equation of $N_{t}$ is as followes.

$$
N_{t}=c_{1} k_{s} a_{s}^{p}
$$

$c_{1}$ represents a coefficient related to cutting edge density of the oilstone, $k_{s}$ represents a coefficient related to cutting edge shape, $p$ is an index. Unit length refers to axial. Dynamic valid cutting edge is less than static valid cutting edge, a less than 1 coefficient $k_{d}$ should be added and its value can be obtained by experiments. The equation of dynamic valid cutting edge numbers is as follows.

$$
N_{d}=k_{d} N_{t} 1_{s} \Delta z
$$

$\Delta z$ represents width of the thin slice, $N_{d}$ represents dynamic valid cutting edge numbers contact with the thin slice which is affected by the diameter of the thin slice.

According to references[6,7], the key parameters in Eq. 5 can be determined as followed: $c_{1}=10^{5} \mathrm{~mm}^{2}, k_{s}=1, k_{d}=0.8, p=1.5, \Delta z=0.1 \mathrm{~mm}$. Fig. 5 shows the result of MATLAB.

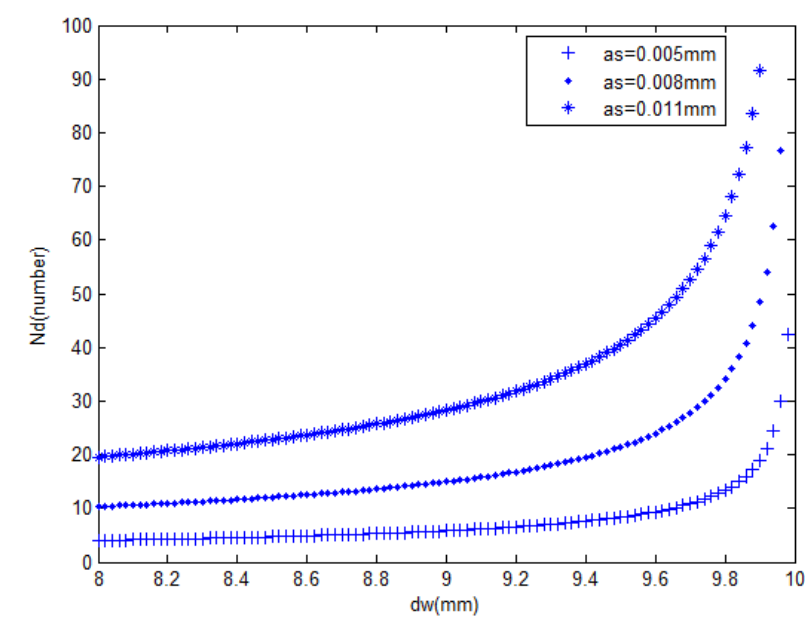

Fig.5 The influence of $a_{s}$ to $N_{d}$

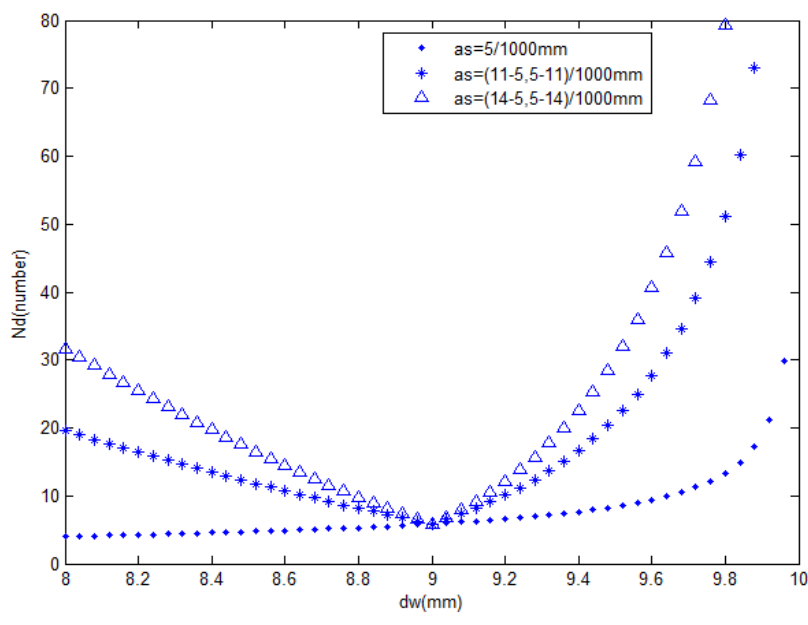

Fig.6 The influence of $a_{s}$ to $N_{d}$

What can be founded by observing the various curves in Fig. 5 is that dynamic valid cutting edge numbers $N_{d}$ increases with the increase of tapered roller diameter $d_{w}$. From the small end of the roller to the middle portion it increases slowly, at the large end of the roller it increases quickly. 
The dynamic valid cutting edge numbers $N_{d}$ increases with the increase of machining surface depth $a_{S}$ when $d_{w}$ is the same, that means $a_{s}$ has a great influence on $N_{d}$. A minor change of $a_{s}$ can change $N_{d}$ significantly and then change roller material removal rate. This conclusion is significant, for the roller material removal rate has a positive correlation with the dynamic valid cutting edge numbers. In the process of superfinishing, to make the roller generatrix obtain the crown, $N_{d}$ must be changed according to certain rules correspondingly. To control $N_{d}$ by changing $a_{s}$ is easier to operate compared with changing other key parameters.

The value of $a_{s}$ should be identified as a variable that it decrease uniformly in the first half and increase uniformly in the second half with increase of $d_{w}$. All the value of parameters are the same as above, Fig. 6 shows the result of MATLAB. $a_{s}$ obtains the minimum value $0.005 \mathrm{~mm}$ when $d_{w}$ equals $9 \mathrm{~mm}$ and obtains the maximum value $0.011 \mathrm{~mm}$ or $0.014 \mathrm{~mm}$ when $d_{w}$ equals $8 \mathrm{~mm}$ or 10mm. Fig. 6 shows that $N_{d}$ obtains the minimum value in the middle part, bigger value in the small end and the maximum in the big end, the curve shape looks like a "V". Symmetric crown of the roller generatrix means that the material removal amount of the middle part is the least, of the small end is bigger and of the big end is the maximum. The shape "V" of $N_{d}$ is perfect.

\section{Summary}

(1) In the superfinishing, the change of radial contact arc length and valid cutting edge numbers is caused by the change of the taper roller diameter.

(2) The arc length and the cutting edge numbers increase slowly in the small end and the middle part but rapidly in the big end with the increase of the roller diameter.

(3) The cutting edge numbers curve shape can be changed by changing cutting surface depth and the ideal curve can be obtained.

\section{Acknowledgements}

This research derives from the National Natural Science Foundation in China (No.U1404517).

\section{References}

[1] Xue Jinxue, Jia Songyang, Yang Baisong: Grinding Analysis of Superfinishing Tapered Roller based on Arc MRR. Coal Mine Machinery. 36-12 (2015), p. 124-126.

[2] Chinese Mechanical Engineering Society, in: Technology Roadmaps of Chinese Rechanical, edtied by Science and technology of China press, Beijing (2011), in press.

[3] Chen Fanghua, Wang Jiugen: Elastohydrodynamic Lubrication of Tapered Roller with Logarithmic Profile. Journal of Mechanical Engineering. 47-19 (2011), p. 143-148.

[4] Ma Jiaju, Xu Wen, Chen Xiaoyang: EHL Properties of Roller Contacts and Its Application, part II: a numerical analysis of EHL for engineering logarithmic roller. Tribology. 20-1 (2000), p. 63-66.

[5] Xue Jinxue, Jia Songyang, Yang Baisong: The Polish Properties Analysis of Through-feed Superfinishing Tapered Roller. Manufacturing Automation. 38-1 (2016), p. 33-37.

[6] Ren J X, Hua D A, in: Principle of Grinding and Cutting, edtied by Publishing house of electronics industry, Beijing (2011), in press.

[7] S-H Chang, T. N Farris and S. Chandrasekar: Experimental Characterization of Superfinishing. Engineering Manufacture. 217 (2003), p. 941-951. 